EDITORA

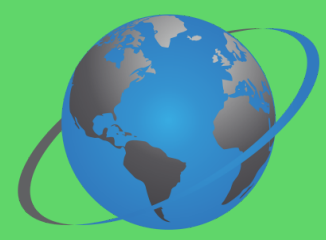

OMNIS SCIENTIA

\title{
SAÚDE PÚBLICA EM
}

\section{TEMPOS PANDEMICOS}

\section{VOLUME 1}

Organizador:

Raul Sousa Andreza

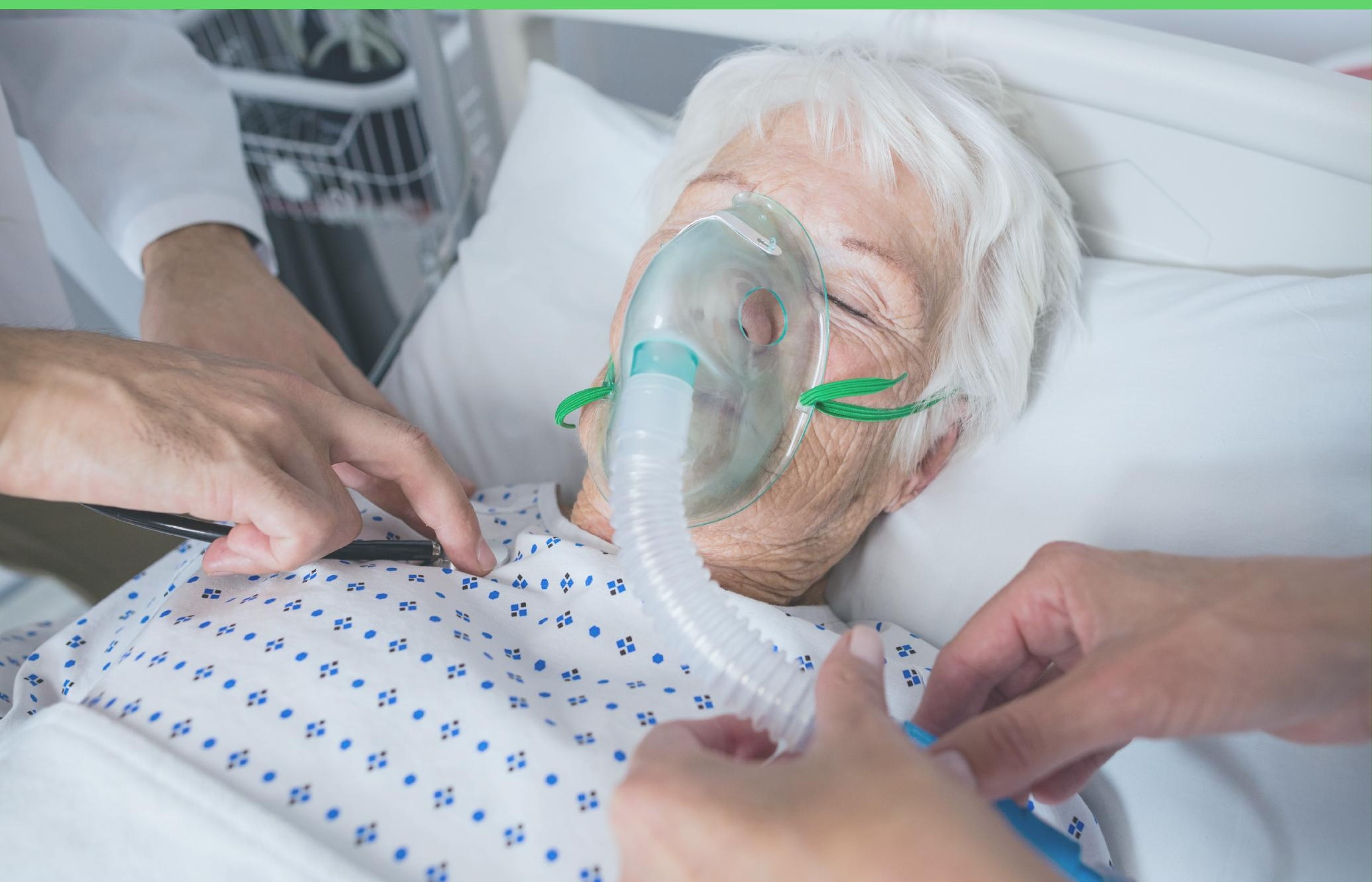


EDITORA

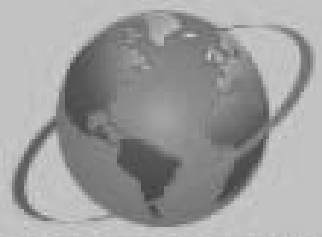

OMNIS SCIENTIA

\section{SAÚDEE PÚBUICA BM}

\section{THEMIPOS PANDENMICOS}

\section{VOLUME 1}

Organizador:

Raul Sousa Andreza

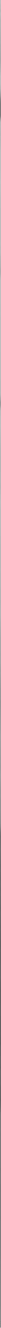


Editora Omnis Scientia

SAÚDE PÚBLICA EM TEMPOS PANDÊMICOS

Volume 1

$1^{a}$ Edição

TRIUNFO - PE

2021 


\section{Editor-Chefe}

Me. Daniel Luís Viana Cruz

Organizador (a)

Prof. Me. Raul Sousa Andreza

\section{Conselho Editorial}

Dra. Pauliana Valéria Machado Galvão

Dr. Wendel José Teles Pontes

Dr. Walter Santos Evangelista Júnior

Dr. Cássio Brancaleone

Dr. Plínio Pereira Gomes Júnior

Editores de Área - Ciências da Saúde

Dra. Camyla Rocha de Carvalho Guedine

Dra. Cristieli Sérgio de Menezes Oliveira

Dr. Leandro dos Santos

Dr. Hugo Barbosa do Nascimento

Dr. Marcio Luiz Lima Taga

Dra. Pauliana Valéria Machado Galvão

\section{Assistentes Editoriais}

Thialla Larangeira Amorim

Andrea Telino Gomes

Imagem de Capa

Freepik

\section{Edição de Arte}

Vileide Vitória Laranjeira Amorim

\section{Revisão}

Os autores

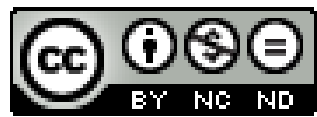

Este trabalho está licenciado com uma Licença Creative Commons - AtribuiçãoNãoComercial-SemDerivações 4.0 Internacional.

O conteúdo abordado nos artigos, seus dados em sua forma, correção e confiabilidade são de responsabilidade exclusiva dos autores. 
Dados Internacionais de Catalogação na Publicação (CIP) (eDOC BRASIL, Belo Horizonte/MG)

S255 Saúde pública em tempos pandêmicos [livro eletrônico] / Organizador Raul Sousa Andreza. - Triunfo, PE: Omnis Scientia, 2021.

286 p. : il.

Formato: PDF

Requisitos de sistema: Adobe Acrobat Reader

Modo de acesso: World Wide Web

Inclui bibliografia

ISBN 978-65-88958-41-4

DOI 10.47094/978-65-88958-41-4

1. Ciências da saúde. 2. Saúde pública. 3. Pandemia. I. Andreza, Raul Sousa.

CDD 610

Elaborado por Maurício Amormino Júnior - CRB6/2422

\author{
Editora Omnis Scientia \\ Triunfo - Pernambuco - Brasil \\ Telefone: +55 (87) 99656-3565 \\ editoraomnisscientia.com.br \\ contato@editoraomnisscientia.com.br
}




\section{PREFÁCIO}

A incidência do novo coronavírus no Brasil é preocupante. No entanto, a saúde pública do país e o sistema de atendimento visa abranger a diversidade que o Brasil apresenta, o sistema único de saúde (SUS) tem como base a integralidade, a universalidade e a equidade de todos os pacientes e trabalhadores. Instituído assim, para democratizar toda a saúde brasileira, tem o interesse de ofertar serviços de qualidade a população. Portanto, ao longo da história de sua consolidação a saúde pública foi deixado de lado e passou a gerar grandes problemas aos profissionais atuantes.

De fato, os estudos desenvolvidos no âmbito da saúde pública se propõem a articular conhecimentos de diferentes saberes e fazeres fornecendo subsídios teóricos, práticos e metodológicos que contribuem positivamente para a construção de estratégias e políticas públicas que visam o desenvolvimento de informações e ações em prol de uma saúde de qualidade para toda comunidade.

O presente livro é composto por 24 capítulos elaborados por autores pertencentes às ciências da saúde e suas áreas afins com o objetivo de proporcionar conhecimentos e compartilhar experiências e resultados de estudos desenvolvidos em várias localidades brasileiras e que visam à elucidação de diferentes situações de saúde.

Em nossos livros selecionamos um dos capítulos para premiação como forma de incentivo para os autores, e entre os excelentes trabalhos selecionados para compor este livro, o premiado foi o capítulo 02, intitulado "VACINAS CONTRA COVID-19: UMA BREVE DESCRIÇÃO POR MEIO DE REVISÃO INTEGRATIVA“. 


\section{SUMÁRIO}

CAPÍTULO 1

A IMPORTÂNCIA E OS DESAFIOS DA ESTRATÉGIA DE SAÚdE DA FAMÍLIA NO ENFRENTAMENTO DA COVID-19

Romana Erica Tavares Grangeiro Pinto

Wyara Ferreira Melo

Maria Amanda Laurentino Freires

Patrício Borges Maracajá

Aline Carla de Medeiros

José Cândido da Silva Nóbrega

Manoel Marques de Souto Nóbrega Filho

Túlio Alberto de Oliveira Sousa

Mônica Valéria Barros Pereira

Vicente Saraiva dos Santos Neto

Hozanna Estrela Celeste

Gabriela Rocha Pordeus dos Santos

DOI: 10.47094/978-65-88958-41-4/17-26

CAPÍTULO 2

VACINAS CONTRA COVID-19: UMA BREVE DESCRIÇÃO POR MEIO DE REVISÃO INTEGRATIVA

Fernanda Lima Marçal

Isabela Figueiredo e Souza

Maria Eduarda Coelho Gomes

Larissa Lima Torres

Isabela Campbell Santos

Thamara Lóren Lima

Ludmilla Vieira Magalhães

Maria Eduarda Sirina Pereira

Lucas Viana de Oliveira 
Larissa da Silva Torres França

Natan Fiorotti da Silva

Milena de Oliveira Simões

DOI: 10.47094/978-65-88958-41-4/27-41

CAPÍTULO 3

AÇÃO EM SAÚdE E A IMPORTÂNCIA DO USO CORRETO DAS MÁSCARAS PARA PREVENÇÃO DE INFECÇÕES RESPIRATÓRIAS

Soniely Nunes de Melo

Rafael Belarmino de Souza Lima

Tarcísio Correia Sposito

Rayana Ribeiro Trajano de Assis

Nayara Sandriele Santana de Souza

Bruna Rafaella Santos Torres

Flávio José Alencar de Melo

Davi Silva de Jesus

Carlos Henrique Bezerra de Siqueira

Izabelle Barbosa da Silva

Marcos André de Holanda Prudente Pessoa

Ana Marlusia Alves Bomfim

DOI: 10.47094/978-65-88958-41-4/42-51

CAPÍTULO 4

CONHECIMENTOS DAS EQUIPES MULTIPROFISSIONAIS DA ESTRATÉGIA DE SAÚDE DA FAMIILIA SOBRE COVID-19 E GESTAÇÃO

Priscilla dos Santos Nascimento

Michelle Araújo Moreira

DOI: 10.47094/978-65-88958-41-4/52-65 
CARTILHA EDUCATIVA SOBRE A COVID-19 PARA A PROTEÇÃO DA COMUNIDADE ACADÊMICA DO CAMPUS BELÉM DO IFPA

Lidineusa Machado Araujo

Maria de Nazaré Pereira Rodrigue Martins

Gabriela Priscila de Lima Carvalho

Fernanda Rafaela de Souza Rebelo da Costa

Michelle da Silva Pereira

Andréa de Melo Valente

Maria Helena Cunha Oliveira

Antônio Marcos Mota Miranda

DOI: 10.47094/978-65-88958-41-4/66-75

CAPÍTULO 6 .76

A SAÚdE DOS PROFISSIONAIS DE SAÚDE QUE ATUAM NA LINHA DE FRENTE NA PANDEMIA DO COVID - 19: REVISÃO INTEGRATIVA

João Lucas Ferreira Andrade

Léa Bianch Lima

Luana Kellen Nogueira Epitácio

Maria Eduarda Alves Vasconcelos

Antônio Augusto Ferreira Carioca

Carlos Antônio Bruno da Silva

Eudóxia Sousa de Alencar

DOI: 10.47094/978-65-88958-41-4/76-89

CAPÍTULO 7 .90

OS EFEITOS DA PANDEMIA NA COMPULSÃO ALIMENTAR: UMA REVISÃO DE LITERATURA

Lais Pontes de Miranda Cerqueira

Tarcio Goncalves sobral

Isadora Bianco Cardoso de Menezes

DOI: 10.47094/978-65-88958-41-4/90-97 
O IMPACTO DA PANDEMIA DO COVID - 19 SOBRE A SEGURANÇA ALIMENTAR E NUTRICIONAL: REVISÃO INTEGRATIVA

Bruna Sousa Barbosa

Igor Matheus Cruz de Oliveira

João Lucas Ferreira Andrade

Léa Bianch Lima

Luana Kellen Nogueira Epitácio

Maria Eduarda Alves Vasconcelos

Antônio Augusto Ferreira Carioca

Carlos Antônio Bruno da Silva

Eudóxia Sousa de Alencar

DOI: 10.47094/978-65-88958-41-4/98-104

CAPÍTULO 9 105

A UTILIZAÇÃO DOS PROTOCOLOS DE USO DO GUIA ALIMENTAR PARA A POPULAÇÃO BRASILEIRA: RELATO DE EXPERIÊNCIA

Bárbara Santos Amorim

Lis Chaves Marinho

Isadora Bianco Cardoso de Menezes

DOI: 10.47094/978-65-88958-41-4/105-114

CAPÍTULO 10 .

O IMPACTO DA COVID-19 EM PORTADORES DE SÍNDROME METABÓLICA

Laura Rasul de Lima

Ana Beatriz Amaral Vieira

Gabriella Neiva Reis

Ingrid Ravenna Liberalino Lima

DOI: 10.47094/978-65-88958-41-4/115-122 
SIMPLIFICANDO A HANSENÍASE: PROJETO DE INTERVENÇÃO REALIZADO EM

MEIO DIGITAL DURANTE A PANDEMIA

Amanda Almeida Lima

Ana Beatriz Sousa Santos

Francisco Vittor Miranda e Araújo

Jesamar Correia Matos Filho

João Ferreira de Paula Neto

Maria Clara de Freita Albano

Manoel Cícero Viana de Lima

Pedro Schmitt Martins Paiva Matos

Ruddy Mariano Maia Cysne Guerra

Samuel Carvalho Vasconcelos

Thaine Mirla Rocha

Elaine Lopes Bomfim

DOI: 10.47094/978-65-88958-41-4/123-131

CAPÍTULO 12.

DESCOMPLICANDO A HANSENÍASE: PROJETO DE INTERVENÇÃO REALIZADO EM UNIDADE DE SAÚDE NO PERÍODO DA PANDEMIA

Adália Stefanny de Araujo Cavalcante

Giovanna Giffoni Souza do Nascimento

Iêda de Freitas Martins Jota

Isabel Camila Araújo Barroso

Kaio Rangel Freitas Guimarães

Laís Mesquita de Sousa

Monique dos Santos Chaves

Manoel Victor Freires Vieira

Matheus Macedo Braga Coelho

Thaine Mirla Rocha

Elaine Lopes Bomfim 
CAPÍTULO 13. 140

HANSENÍASE: UM ESTUDO EPIDEMIOLÓGICO NA REGIÃO DO MÉDIO ARAGUAIAMATO GROSSO

Flavia Rodrigues Santana

Josilene Dália Alves

DOI: 10.47094/978-65-88958-41-4/140-150

CAPÍTULO 14 151

MUNICÍPIOS COM ALTA INCIDÊNCIA DE TUBERCULOSE EM MATO GROSSO: CONHECER PARA INTERVIR EM TEMPOS DE PANDEMIA

Larissa Machado Bellé

Yasmim Paloma Abreu Silva

Alessandro Rolim Scholze

Josilene Dália Alves

DOI: 10.47094/978-65-88958-41-4/151-162

CAPÍTULO 15 163

SAÚDE INTEGRAL DA MULHER EM CONTEXTO PANDÊMICO: REVISÃO INTEGRATIVA DE LITERATURA

Lohana Guimarães Souza

Tailande Venceslau Carneiro

Letícia Grazielle Santos

DOI: 10.47094/978-65-88958-41-4/163-175

CAPÍTULO 16 176

ASSISTÊNCIA DE ENFERMAGEM NO OUTUBRO ROSA E PERFIL EPIDEMIOLÓGICO DAS MULHERES: RELATO DE EXPERIÊNCIA

Helena Pereira de Souza

Laura Letícia Perdição Guerra

Luana Fernandes e Silva 
Thales Philipe Rodrigues da Silva

Alessandra Lage Faria

Helen Carine Ferreira Balena

Érica Moreira de Souza

Bruna Luíza Soares Pinheiro

Lorena Medeiros de Almeida Mateus

Flávia Duarte de Oliveira Ribeito

Bianca Maria Oliveira Luvisaro

Fernanda Penido Matozinhos

DOI: 10.47094/978-65-88958-41-4/176-188

CAPÍTULO 17 189

AGRAVAMENTO DAS DOENÇAS PSIQUIÁTRICAS DURANTE O PERÍODO DE ISOLAMENTO SOCIAL: UMA BREVE REVISÃO DE LITERATURA

Hellen Kristina Magalhães Brito

Gabriela Teixeira Lima

Ana Laura Fernandes Tosta

Laura Beatriz Caitano de Oliveira

Maria Paula Ricardo Silva

Mariana Vieira Garcia de Carvalho

Nathália Siriano Costa

Mayara Rita Figueredo

Mabel Fernandes Rocha

Helena Maria Mendes Marques

Kaio Murilo Santana Corrêa

Ana Flávia Buiatte Andrade

DOI: 10.47094/978-65-88958-41-4/189-200 
ATUAÇÃO DO PSICÓLOGO EM TEMPO DE PANDEMIA: UMA REVISÃO DE ESTUDOS NACIONAIS

Gabriel Rigamonte

Sueli Souza

Wilson Quiroz

Daniel Bartholomeu

Fernando Pessotto

Cintia Heloína Bueno

Fernanda Helena Viana Garcia

DOI: 10.47094/978-65-88958-41-4/201-213

CAPÍTULO 19

CRIAÇÃO DE UM APLICATIVO VOLTADO PARA UMA UNIDADE BÁSICA DE SAÚdE EM FORTALEZA

Isabella Araujo Duarte

Giovanna Rolim Pinheiro Lima

Idna Lara Goes de Sena

Laura Figueiredo Leite

Letícia Cavalcante Lócio

Livian Araújo Camelo Gomes

Maria Regina Cardoso Linhares Oliveira Lima

Maria Tereza Linhares Cardoso

Pedro Henrique Cardoso Nogueira

Rafael Albuquerque Franco

Rodrigo Carvalho Paiva

Berta Augusta Faraday Sousa Pinheiro

DOI: 10.47094/978-65-88958-41-4/214-229 
TELEMEDICINA E SUAS VARIÁVEIS NO CENÁRIO DE PANDEMIA MUNDIAL: UMA REVISÃO DE LITERATURA

Raniere Canteiro Garcia Lhamas

Andressa Marcolino Campos

Douglas Ferreira Lima

Gabriel Souza Ferreira Oliveira

Guilherme de Mendonça Lopes Beltrão

Luciana de Paula Santana

Nícollas Nunes Rabelo

DOI: 10.47094/978-65-88958-41-4/230-237

CAPÍTULO 21 238

MONITORIA ACADÊMICA NO ENSINO REMOTO: PERSPECTIVAS E DESAFIOS EM TEMPOS PANDÊMICOS

Felipe Gabriel Assunção Cruz

Givanildo Bezerra de Oliveira

Marcílio Delan Baliza Fernandes

Ana Lúcia Moreno Amor

DOI: 10.47094/978-65-88958-41-4/238-248

CAPÍTULO 22

A DOENÇA DO OLHO SECO NA SÍINDROME DE SJÖGREN

Bruna Rafaella Santos Torres

Carlos Eduardo Ximenes da Cunha

Carlos Henrique Bezerra de Siqueira

Flavia Emanuelly Alves França Gomes

Santília Tavares Ribeiro de Castro e Silva

Anna Caroline Guimarães Gomes

Laís Rytholz Castro

Dennis Cavalcanti Ribeiro Filho 
Lara Medeiros Pirauá de Brito

Marina Viegas Rezende Ribeiro

DOI: 10.47094/978-65-88958-41-4/249-259

CAPÍTULO 23 260

FEIRAAGROECOLÓGICA: DIFICULDADES E POTENCIALIDADES DE UM CIRCUITO CURTO DE COMERCIALIZAÇÃO

Maria Rita Garcia de Medeiros

Rônisson Thomas de Oliveira Silva

Maria Natalícia de Lima

Ana Beatriz Macêdo Venâncio dos Santos

DOI: 10.47094/978-65-88958-41-4/260-269

CAPÍTULO 24 270

AVALIAÇÃO DO IMPACTO DE UMA REDE SOCIAL EM USUÁRIOS DE UMA UNIDADE BÁSICA DE SAÚDE EM FORTALEZA

Luiz Gerson Gonçalves Neto

Letícia Cavalcante Lócio

Carlos Alexandre Leite Pereira Filho

Henrique Sousa Costa

Maria Helena dos Santos Macedo

Lígia Bringel Olinda Alencar

Berta Augusta Faraday Sousa Pinheiro

Isaac Dantas Sales Pimentel

DOI: 10.47094/978-65-88958-41-4/270-280 
Soniely Nunes de Melo';

Centro Universitário Tiradentes - UNIT AL, Maceió, Alagoas.

http://lattes.cnpq.br/5078713169675908

Rafael Belarmino de Souza Lima²;

Centro Universitário Tiradentes - UNIT AL, Maceió, Alagoas.

http://lattes.cnpq.br/3658453904413896

Tarcísio Correia Sposito $^{3}$;

Centro Universitário Tiradentes - UNIT AL, Maceió, Alagoas.

https://orcid.org/0000-0002-8963-0556

Rayana Ribeiro Trajano de Assis ${ }^{4}$;

Centro Universitário Tiradentes - UNIT AL, Maceió, Alagoas.

http://lattes.cnpq.br/1317002328649637

Nayara Sandriele Santana de Souza ${ }^{5}$;

Centro Universitário Tiradentes - UNIT AL, Maceió, Alagoas.

http://lattes.cnpq.br/6893851063509658

Bruna Rafaella Santos Torres ${ }^{6}$;

Centro Universitário Tiradentes - UNIT AL, Maceió, Alagoas.

http://lattes.cnpq.br/9556991601884243

Flávio José Alencar de $\mathrm{Melo}^{7}$;

Centro Universitário Tiradentes - UNIT AL, Maceió, Alagoas.

http://lattes.cnpq.br/3283758437904102

Davi Silva de Jesus ${ }^{8}$;

Centro Universitário Tiradentes - UNIT AL, Maceió, Alagoas.

http://lattes.cnpq.br/4535592678465360

Carlos Henrique Bezerra de Siqueira';

Centro Universitário Tiradentes - UNIT AL, Maceió, Alagoas.

http://lattes.cnpq.br/0020231225282733 


\section{Izabelle Barbosa da Silva ${ }^{10}$;}

Centro Universitário Tiradentes - UNIT AL, Maceió, Alagoas.

http://lattes.cnpq.br/2742064862005163

Marcos André de Holanda Prudente Pessoa';

Centro Universitário Tiradentes - UNIT AL, Maceió, Alagoas.

http://lattes.cnpq.br/7040541342436074

Ana Marlusia Alves Bomfim ${ }^{12}$.

Centro Universitário Tiradentes - UNIT AL, Maceió, Alagoas.

http://lattes.cnpq.br/2659414598724448

RESUMO: Introdução: As infecções respiratórias virais são prevalentes e de alta transmissibilidade. Prova disso é a covid-19, doença causada pelo coronavírus SARS-Cov-2, e transmitida principalmente por gotículas expelidas por pessoas infectadas pelo vírus. Por isso, máscaras de tecido reduzem a transmissão de doenças e podem ser eficazes na redução da contaminação. Assim, é fundamental a divulgação do seu uso e manuseio correto por meio de atividades de promoção em saúde para a população, onde o escasso acesso a informações pertinentes pode comprometer sua efetividade. Objetivo: Educar a população sobre o uso das máscaras, sua correta execução e manutenção adequada. Metodologia: Trata-se de estudo qualitativo, descritivo e exploratório, do tipo pesquisa-intervenção. Com base na fundamentação teórica encontrada nas bases de dados Scielo, Pubmed e LILACS foi confeccionado um folder digital sobre a forma correta de usar, preservar e escolher as máscaras de tecido utilizadas pela população. A divulgação do folder ocorreu nas redes sociais "Whatsapp" e "Instagram" com amplo compartilhamento entre os jovens e adultos com acesso às redes sociais. Resultados e Discussão: Identificou-se que a disseminação do material digital proposto atingiu parcela significativa da população com acesso às redes sociais e provocou uma discussão coletiva. Outrossim, foi perceptível a abrangência de diferentes níveis socioeconômicos e culturais, ficando evidente a sua contribuição para o maior entendimento da prevenção de doenças. Ainda, verificou-se déficit no conhecimento a respeito da individualização dos equipamentos de proteção. Conclusão: Diante do exposto, observa-se que a utilização das máscaras consiste em instrumento importante no combate a infecções respiratórias, como a covid-19, sendo notório déficit no manejo ideal desse instrumento de prevenção. Com isso, iniciativas de educação em saúde e de conscientização acerca do uso correto das máscaras de proteção são medidas fundamentais para reduzir a propagação do vírus.

PALAVRAS-CHAVE: COVID-19. Máscaras Faciais. Educação em Saúde. 
ABSTRACT: Introduction: Viral respiratory infections are prevalent and highly transmissible, like covid-19, a disease caused by the SARS-Cov-2 coronavirus, and transmitted mainly by droplets expelled by people infected. Therefore, cloth masks reduce the transmission of disease and can be effective in reducing contamination. Thus, it is essential to disseminate its use and correct handling through health promotion activities for the population, where the scarce access of information can compromise its effectiveness. Objective: Educate the population about the use of masks, their correct execution and proper maintenance. Methodology: This is a qualitative, descriptive and exploratory study, of the research-intervention type. Based on the theoretical foundation found on Scielo, Pubmed and LILACS databases, a digital folder was created on the correct way to use, preserve and choose the fabric masks used by the population. The folder was disseminated on social media like "Whatsapp" and "Instagram" with wide sharing among young people and adults. Results and Discussion: It was identified that the dissemination of the proposed digital material reached a significant portion of the population with access to social media and provoked a collective discussion. Furthermore, the range of different socioeconomic and cultural levels was noticeable, making its contribution to a greater understanding of disease prevention evident. Still, there was a deficit in knowledge regarding the individualization of protective equipment. Conclusion: Given the above, it is observed that the use of masks is an important instrument in the fight against respiratory infections, such as covid-19, with a notable deficit in the ideal management of this prevention instrument. As a result, health education and awareness initiatives about the correct use of protective masks are fundamental measures to reduce the spread of the virus.

KEY-WORDS: COVID-19. Facial Masks. Health Education.

\section{INTRODUÇÃO}

As infecções respiratórias virais têm alta prevalência devido à diversidade de agentes infecciosos e de sorotipos, estes associados à elevada capacidade de transmissibilidade entre as pessoas. Prova disso é a covid-19, doença causada pelo coronavírus SARS-Cov-2, e transmitida principalmente por meio do contato com pequenas gotículas que contêm o vírus e são expelidas por pessoas infectadas. Em contato com a via aérea, o vírus se multiplica e aumenta a capacidade viral de infectar as células do nosso organismo.

As transmissões aéreas, por meio de gotículas, e por contato físico são as principais rotas de contaminação dos vírus respiratórios, sendo propícias em circunstâncias e ambientes específicos nos quais os procedimentos de geração de aerossol (AGP) são realizados. Aerossóis são partículas líquidas dispersas no ar que podem conter microrganismos ou substâncias industriais. 
Isso justifica a hipótese de que quando uma pessoa infectada com SARS-CoV-2 respira pesadamente, espirra ou tosse, o SARS-CoV-2 será excretado e transformado em bioaerossóis (TABATABAIZADEH, 2021).

Com isso, a máscara é uma forma de reduzir o contato direto com a carga viral exposta como uma barreira direta contra o agente infeccioso. Dessa forma, descobriu-se que a eficácia do uso de máscaras pela comunidade está relacionada com a redução do risco de infecção clínica, evitando a inalação de patógenos e reduzindo o contato face a face (MACINTYRE, et al., 2009). Máscaras de tecido, como as de algodão com alta densidade de fios, reduzem a transmissão de gotículas e assim, podem ser eficazes na redução da contaminação pelo SARS-CoV-2. Em situações de baixo risco, o uso de máscaras de tecido de múltiplas camadas fornece proteção contra a transmissão da covid-19. Entretanto, a influência da umidade produzida pela expiração, o tempo de uso e o número de lavagens são fatores que podem reduzir sua eficácia (SILVA. et al., 2020).

Sendo assim, é de suma importância o uso correto da mesma, bem como da realização de sua manutenção rotineira, visto que, por conta do contato direto ao meio externo ela pode se tornar um meio propício para autocontaminação, desse modo, deve ser fundamental a ampla divulgação do uso correto e manuseio da mesma por meio de atividade de promoção em saúde na população em geral, onde o escasso acesso a informações pertinentes pode comprometer a efetividade desejada da máscara para proteção.

A partir disso, esse estudo objetivou educar a população em geral sobre os benefícios do uso das máscaras, sua correta execução e manutenção adequada.

\section{METODOLOGIA}

Trata-se de estudo qualitativo, exploratório e descritivo, do tipo pesquisa-intervenção. Sabendo a importância do autocuidado e do uso correto das máscaras para o enfrentamento da pandemia do vírus SARS-CoV-2, foi confeccionado um folder digital sobre a forma correta de usar, preservar e escolher as máscaras de tecido utilizadas pela população geral, a partir de fundamentação teórica encontrada nas bases de dados Scielo, Pubmed e LILACS.

O folder (figura 1) possui título "a minha te protege e a sua me protege" e contém imagens ilustrativas acompanhadas de texto sobre a lavagem das mãos antes e após o manejo das máscaras, a retirada e posicionamento correto da mesma a fim de cobrir nariz, boca e queixo e evitar espaços nas laterais, bem como esclarecimento de erros comuns, como o compartilhamento de máscaras, o período de uso diário, validade e número de camadas. A linguagem utilizada foi direta e destinada a população em geral, sem utilização de jargões e com enfoque no entendimento do leitor. Depois de finalizado, o folder foi divulgado nas redes sociais Whatsapp e Instagram para amplo compartilhamento e interação com a mídia produzida. Abaixo a figura descreve o folder elaborado e utilizado para a ação remota. 
Figura 1: Folder "A minha te protege e a sua me protege" - instruções acerca do uso correto das máscaras de tecido faciais.

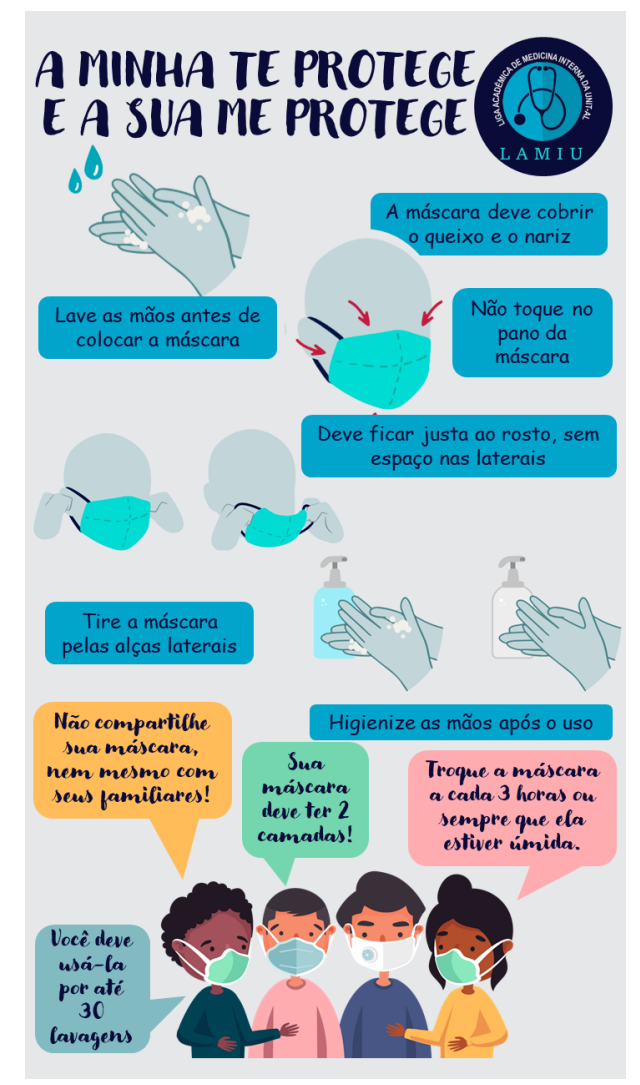

Fonte: Produção pessoal. 2021.

\section{RESULTADOS E DISCUSSÕES}

A partir da atividade realizada, foi possível identificar que a disseminação do material digital proposto, atingiu parcela significativa da população com acesso às redes sociais e provocou uma discussão coletiva, especialmente nas multiplataformas gratuitas de mensagens instantâneas, Whatsapp e Instagram. Através da interação e da devolutiva do público alvo, foi perceptível que essa ação em saúde abrangeu diferentes níveis socioeconômicos e culturais, ficando evidente a sua contribuição para o maior entendimento do uso correto das máscaras de tecido por parte deste.

Nessa perspectiva, a ação e promoção da saúde baseiam-se na capacidade da comunidade de se identificar como agente ativo na gestão dos riscos aos quais está submetida. Este conceito baseia-se no pressuposto iluminista de Spink, o qual afirma que por sermos seres racionais passíveis de tomada de decisão, a disponibilidade de informação nos torna parceiros da gestão dos riscos (SPINK, 2009). Consequentemente, os materiais digitais fomentam o modelo de atenção e saúde e basta expressar medidas, que a população buscará, automaticamente, formas para se prevenir (MARTINS; SPINK, 2019).

Em consonância, a educação em saúde representa um dos principais elementos para a promoção à saúde, e é uma forma de cuidar que leva ao desenvolvimento de uma consciência crítica, reflexiva e para a emancipação dos sujeitos, pois possibilita que as pessoas passem a cuidar melhor de si mesmas e de seus familiares (SANTOS; PENNA, 2009 e SANTOS et al., 2020). 
Outrossim, a promoção à saúde deve ser relacionada com o contexto em que se vive, com práticas pautadas na visão holística do sujeito, na melhoria da qualidade de vida física, psíquica e espiritual, sendo o profissional e os universitários da área da saúde, atores nesse processo (DIAS et al., 2018).

Portanto, diante do cenário atual da pandemia do Covid-19, as medidas de proteção individual, destacando-se o uso de máscaras, representam o equipamento de proteção respiratória indicado para controlar as exposições a gotículas de saliva, com o objetivo de reduzir o risco da transmissão da Covid-19. Os principais benefícios de usar uma máscara incluem limitar a propagação do vírus de alguém que sabe ou não sabe que tem uma infecção para outras pessoas, assim como, reforçar a necessidade de continuar a prática do distanciamento físico (DESAI e ARONOFF, 2020). Entretanto, a Organização Mundial da Saúde (OMS) destaca alguns potenciais riscos causados pelo mau uso da máscara, como as chances de autocontaminação através de uma má manipulação da mesma, assim como à contaminação pela falta de troca quando necessária, além disso, pode passar uma falsa sensação de segurança, diminuindo outras medidas higiênicas (OMS, 2020).

Diante da emergência em propor medidas alternativas e seguras, as máscaras de tecido surgem como forma de proteção de baixo custo, rápida produção e impede o uso indiscriminado de máscaras cirúrgicas, as quais devem ser priorizadas para fins hospitalares, onde são de maior necessidade (LIMA, et al., 2020).

As máscaras de tecido são vistas como equipamentos de proteção de uso não profissional, indicadas para uso pela população em geral, como uma barreira física para reduzir a disseminação de gotículas e secreções expelidas pelo nariz e/ou boca do usuário no ambiente. (ANVISA, 2020).

Embora as máscaras de tecido tenham eficácia reduzida ao serem comparadas com máscaras hospitalares (N95 e/ou máscara cirúrgica), quando fabricadas com duplas camadas, podem ser tão eficazes quanto. Acerca disso, estudo realizado demonstrou que, quando usados em camadas duplas, os têxteis são tão eficazes quanto os materiais de máscara/tecido cirúrgico na redução da dispersão de gotículas para $<10 \mathrm{~cm}$ e a área de contaminação circunferencial para $\sim 0,3 \%$ (RODRIGUEZPALACIOS, et al.,2020).

É válido ressaltar que a eficácia da barreira das máscaras contra as gotículas é influenciada, não apenas pela quantidade de camadas, mas também e principalmente, pelo tipo de tecido e frequência de lavagens.

Quanto aos tipos de tecidos, foi destacada a utilização de tecidos com menor porosidade, além de tecidos que atuam como filtro eletrostático de partículas (por exemplo: seda, chiffon e flanela) (CHU, et al., 2020). Quanto às recomendações do Ministério da Saúde recomenda em ordem decrescente de capacidade de filtragem: 1) saco de aspirador, 2) cotton (composto de poliéster 55\% e algodão 45\%), 3) algodão e 4) fronhas de tecido antimicrobiano (BRASIL, 2020 e GIRARDI et al., 2020). 
No tocante à frequência das lavagens, a pesquisa realizada no Nepal evidenciou que a eficácia das máscaras de tecido é reduzida 20\% após a quarta lavagem e secagem (NEUPANE et al., 2019). Essa perda de eficácia na filtração de partículas ocorre porque o processo de higienização reduz as microfibras do tecido e aumenta o tamanho dos poros (LIMA et al., 2020).

As recomendações da Agência Nacional de Vigilância Sanitária (ANVISA), por sua vez, indicam até trinta ciclos de lavagem (ANVISA, 2020). Destaca-se que a OMS recomenda os cuidados com as máscaras de tecido, porém, não delimita a quantidade de lavagens e o Ministério da Saúde recomenda a troca da máscara após sinais de desgastes (OMS, 2020 e LIMA et al., 2020).

Salienta-se a importância de informar a comunidade acerca da higienização da máscara após sua utilização, conforme orientações da OMS: 1) lavar a máscara em água quente, com sabão ou detergente utilizados para roupas por um minuto; ou 2) colocar a máscara submersa em cloro a $0,1 \%$ por um minuto e lavar abundantemente com água em temperatura ambiente, para eliminar resíduo tóxico do cloro (OMS, 2020).

Com base na interação gerada com público-alvo durante a discussão realizada de forma remota, WhatsApp e Instagram, identificou-se um déficit no conhecimento a respeito da individualização do uso das máscaras, uma vez que muitos não tinham consciência de que as máscaras não devem ser compartilhadas nem mesmo com os familiares. As máscaras também precisam ser trocadas a cada três horas ou se estiverem molhadas ou visivelmente sujas (OMS, 2020).

Ademais, a população precisa estar ciente de que independentemente da capacidade de filtragem ou vedação da máscara, uma vez que a doença possui mais de uma via de transmissão, é fundamental a adoção de medidas preventivas adicionais, tais como o isolamento de casos, medidas de etiqueta respiratórias, higiene das mãos e distanciamento social (CHU, et al., 2020).

Diante dos fatos mencionados, é sabido que o mascaramento universal em público complementa o distanciamento social e a higiene das mãos ao conter ou desacelerar o crescimento exponencial da pandemia (ESPOSITO et al., 2020).

Assim, torna-se evidente a necessidade de maior engajamento dos profissionais e acadêmicos da área da saúde para a orientação da população nas mídias sociais sobre o uso e a lavagem corretos das máscaras de tecido com o intuito de otimizar a eficácia protetora deste utensílio por um período mais prolongado (LIMA, et al., 2020).

\section{CONCLUSÃO}

Diante do exposto, observa-se que a utilização das máscaras faciais consiste em um instrumento importante no combate a infecções respiratórias virais, como a COVID-19, porém mesmo um ano após o início da pandemia é notório que boa parte da população ainda não tem conhecimento suficiente para utilizar de forma eficaz as máscaras de tecido. Iniciativas de educação em saúde e de conscientização acerca do uso correto das máscaras de proteção são medidas fundamentais para reduzir a propagação do vírus e, portanto, devem ser vistas como responsabilidade social de todos os indivíduos. Considerando ainda que a pandemia da covid-19 a cada dia atinge números alarmantes 
de contaminação, pode-se concluir que as máscaras estarão presentes na rotina das pessoas por um longo período, sendo fundamental o conhecimento sobre a sua correta utilização. Foi evidente que as informações passadas durante a ação em saúde impactaram de forma positiva na população alvo, fazendo com que as pessoas refletissem a respeito do uso correto das máscaras. Ademais, os acadêmicos participantes puderam observar as principais dúvidas da população acerca do tema tendo a possibilidade de solucioná-las por meio das redes sociais, além da contribuição para a humanização dos futuros profissionais da área da saúde. Portanto, vale ressaltar a importância de orientar a população com informações seguras e baseadas na ciência, de forma clara e objetiva, para diminuir as chances de surgimento de novas variantes e o colapso do sistema público de saúde.

\section{DECLARAÇÃO DE INTERESSES}

Nós, autores deste artigo, declaramos que não possuímos conflitos de interesses de ordem financeira, comercial, político, acadêmico e pessoal.

\section{REFERÊNCIAS}

ANVISA. Instrução Normativa de abril/2020. ORIENTAÇÕES GERAIS: Máscaras faciais de uso não profissional, [S. l.]: Ministério da Saúde, p. 2-9, 3 abr. 2020. Disponível em: https://www.gov. br/defesa/pt-br/assuntos/desporto-militar/sua-saude/orientacoes-gerais-mascaras-faciais-de-uso-naoprofissional/NTMascarasTecidoAnvisa.pdf. Acesso em: 4 maio 2021.

ANVISA. RESOLUÇÃO no 356, de 23 de março de 2020. Dispõe, de forma extraordinária e temporária, sobre os requisitos para a fabricação, importação e aquisição de dispositivos médicos identificados como prioritários para uso em serviços de saúde, em virtude da emergência de saúde pública internacional relacionada ao SARS-CoV-2. RESOLUÇÃO - RDC No 356, DE 23 DE MARÇO DE 2020, DIÁRIO OFICIAL DA UNIÃO, v. 56-C, p. 5, 23 mar. 2020. Disponível em: https://www. in.gov.br/en/web/dou/-/resolucao-rdc-n-356-de-23-de-marco-de-2020-249317437. Acesso em: 3 maio 2021.

CHU, D.K. et al. Physical distancing, face masks, and eye protection to prevent person-to-person transmission of SARS-CoV-2 and COVID-19: a systematic review and meta-analysis. THE LANCET, [s. l.], v. 395, p. 1-15, 1 jun. 2020. Disponível em: https://doi.org/10.1016/S0140-6736(20)31142-9. Acesso em: 3 maio 2021.

CZERESNIA, D.; FREITAS, C.M. Promoção da Saúde: conceitos, reflexões, tendências.. 2. ed. rev. e aum. Rio de Janeiro: Fiocruz, 2017. 176 p. ISBN 978-85-7541-183-4.

DIAS, I.K.R. et al. Nursing educators' perceptions of the domains of the core competencies framework for health promotion. Cogitare Enferm, v.23, n.2, p.e52664. 2018. Disponível em: https://doaj.org/ article/08f7dca3d7e341e78e4a10a4b16e45e0 Acesso em 01 maio 2021.

ESPOSITO, S. et al. Universal use of face masks for success against COVID-19: evidence and 
implications for prevention policies. European Respiratory Journal: Flagship Scientific Journal of ERS, PubMed, v. 55, ed. 6, 18 jun. 2020. Disponível em: https://doi.org/10.1183/13993003.012602020. Acesso em: 4 maio 2021.

LIMA, C.A. et al. The theory into practice: teaching-service dialogue in the context of primary healthcare in the training of nurses. Rev Fund Care, v.8, n.4, p.5002-9. 2016. Disponível em: https:// www.ssoar.info/ssoar/handle/document/53651. Acesso em: 01 maio 2021.

LIMA, M.M.S. et al. Cloth face masks to prevent Covid-19 and other respiratory infections. Rev. Latino-Am. Enfermagem, Ribeirão Preto, v. 28, 10 ago. 2020. Disponível em: https://doi. org/10.1590/1518-8345.4537.3353. Acesso em: 4 maio 2021.

MARTINS, M.H.M.; SPINK, M.J.P. Comunicação em saúde nas campanhas de prevenção à leptospirose humana em Maceió, Alagoas, Brasil. INTERFACE: Comunicação, Saúde, Educação, Botucatu, v. 23, 26 ago. 2019. Disponível em: https://doi.org/10.1590/interface.180709. Acesso em: 3 maio 2021.

MINISTÉRIO DA SAÚDE. GIRARDI, J.M. et al. INFORME TÉCNICO de agosto/2020. O uso de máscaras para a prevenção e controle da COVID-19, Brasília: Fiocruz, p. 1-8, ago 2020. Disponível em: https://www.arca.fiocruz.br/bitstream/icict/42560/4/Informe\%20Uso_M\%c3\%a1scaras_\%20 final\%20PDF.pdf. Acesso em: 3 maio 2021.

MINISTÉRIO DA SAÚDE. Nota Informativa $\mathrm{N}^{\circ} 03$ de 2020. NOTA INFORMATIVA N 3/2020-CGGAP/DESF/SAPS/MS, Brasil: Ministério da Saúde, n. 03, 2020. Disponível em: http:// portal.antaq.gov.br/wp-content/uploads/2020/04/1586014047102-Nota-Informativa.pdf. Acesso em: 1 maio 2021.

NEUPANE, B.B. et al. Optical microscopic study of surface morphology and filtering efficiency of face masks. PeerJ: Life \& Environment, [s. l.], ed. 7, 17 maio 2019. Disponível em: https://dx.doi. org/10.7717\%2Fpeerj.7142. Acesso em: 3 maio 2021.

ORGANIZAÇÃO MUNDIAL DA SAÚDE. Instrução normativa de 05 de junho de 2020. Recomendações sobre o uso de máscaras no contexto da COVID-19: interim guidance, [S. l.]: World Health Organization, p. 1-17, 5 jun. 2020. Disponível em: https://apps.who.int/iris/ handle/10665/332293. Acesso em: 1 maio 2021.

RODRIGUEZ-PALACIOS, A. et al. Textile Masks and Surface Covers: A 'Universal Droplet Reduction Model' Against Respiratory Pandemics. Medrxiv: The preprint server for health sciences, [s. l.], p. 1-13, 10 abr. 2020. Disponível em: https://doi.org/10.1101/2020.04.07.20045617. Acesso em: 3 maio 2021.

SANTOS, R.V.; PENNA, C.M.M. A educação em saúde como estratégia para o cuidado à gestante, puérpera e ao recém-nascido. Texto Contexto Enferm, v.18, n.4, p.652-60. 2009. Disponível em: https://www.scielo.br/scielo.php?pid=S0104-07072009000400006\&script=sci_abstract\&tlng=pt . Acesso em 01 maio 2021. 
SANTOS, L.C.B. et al. Atividades de Promoção da Saúde Desempenhadas pelo Enfermeiro Atuante na Atenção Primária à Saúde. In: ARRUDA, E.F.; DIÓGENES, B.S. Saúde Pública no Século XXI. 1. ed. Triunfo-PE: Omnis Scientia, 2021. v. 1, cap. 3, p. 42-53. ISBN 978-65-88958-30-8. Disponível em: https://editoraomnisscientia.com.br/editora/artigoPDF/475234801.pdf. Acesso em: 3 maio 2021.

SILVA, N.C.C. et al. Knowledge and health promotion practice of Family Health Strategy nurses. Rev. Bras. Enferm, v.73, n.5, p.e20190362. 2020. Disponível em: https://www.scielo.br/pdf/reben/ v73n5/0034-7167-reben-73-05-e20190362.pdf . Acesso em maio 2021.

SILVA, R.S.M. et al. Uso de máscara de tecido pela população na contenção da disseminação da COVID-19: scoping review. Comunicação em Ciências da Saúde, [s. l.], ed. 31, p. 162-183, 2020. Disponível em: http://www.escs.edu.br/revistaccs/index.php/comunicacaoemcienciasdasaude/article/ view/730/315. Acesso em: 3 maio 2021.

SPINK, M.J.P. Estilos de vida saudável e práticas de existência: fronteiras e conflitos. In: BERNARDES, J.; MEDRADO, B. PSICOLOGIA SOCIAL E POLÍTICAS DE EXISTÊNCIA: fronteiras e conflitos. 1. ed. Maceió-AL: ABRAPSO, 2009. cap. 1, p. 15-26. ISBN 978-85-86472-13-8. Disponível em: https://www.abrapso.org.br/download/download?ID_DOWNLOAD=561. Acesso em: 3 maio 2021.

TABATABAEIZADEH, S.A. Airborne transmission of COVID-19 and the role of face mask to prevent it: a systematic review and meta-analysis. European Journal of Medical Research, [s. l.], v. 26, 2 jan. 2021. Disponível em: https://doi.org/10.1186/s40001-020-00475-6. Acesso em: 1 maio 2021.

WANG, J. et al. Mask use during COVID-19: A risk adjusted strategy. Environmental Pollution, ELSEVIER, v. 266, n. 1, 25 jun. 2020. Disponível em: https://doi.org/10.1016/j.envpol.2020.115099. Acesso em: 4 maio 2021.

DESAI, Angel N.; ARONOFF, David M.. Masks and Coronavirus Disease 2019 (COVID-19). Jama, [S.L.], v. 323, n. 20, p. 2103, 26 maio de 2020. American Medical Association (AMA). http://dx.doi. org/10.1001/jama.2020.6437. 


\section{Índice Remissivo}

$\mathbf{A}$

Acesso aos psicólogos 201, 203, 204

Acesso às redes sociais 43

Agricultor e o consumidor 260

Agricultores familiares 260, 263, 266

Alterações do metabolismo 115

Ambiente virtual 215

Ansiedade 77, 78, 81, 82, 83, 84, 86, 87, 91, 92, 94, 95, 117, 121, 190, 192, 193, 194, 197, 204, 207, 208, 209

Aplicativo 136, 215, 217, 218, 219, 220, 221, 222, 223, 224, 225, 226, 227, 228, 243, 271, 272, 273, 274, 278, 280

Aprendizagem 239

Assistência social 163, 171, 205

Associação da covid-19 com a gestação 52

Astrazeneca (universidade de oxford) 28, 30

Atenção primária 25, 124

Atenção primária à saúde (aps) 19, 59, 107, 125, 177, 178

Atendimento ambulatorial 105

Atendimento on-line 201, 203

Atendimento remoto 202, 205

Atividades econômicas 28

Autoanticorpos 250

Autoridades sanitárias 67, 69, 73, 278

C

Capacidade de defesa do organismo 115

Características dos imunizantes 28, 30

Cartilha educativa sobre a covid-19 67, 69, 70

Ceratoconjuntivite sicca $(\mathrm{kcs}) 250$

Combate à hanseníase 133

Comercialização 38, 260, 261, 262, 263, 264, 265, 266, 267, 268, 269

Compulsão alimentar 90, 92, 93, 94, 95, 96

Consumo de alimentos 105

Contaminação 43, 44, 45, 47, 49, 72, 73, 74, 78, 117, 135, 179, 191, 193, 195, 197

Coronavac (sinovac) 28, 29

Cuidados individuais e coletivos $67,69,73$

Cuidados preventivos ao covid-19 115

D

Depressão 78, 81, 82, 83, 87, 91, 92, 94, 95, 110, 172, 190, 192, 193, 194, 197, 207, 208, 209

Desemprego 21, 99, 103, 153

Desenvolvimento do câncer 177, 185

Desigualdade em saúde 163

Desinformação 29, 208 
Diabetes 21, 56, 57, 95, 105, 106, 107, 108, 114, 116, 117, 118, 119, 120, 181, 183, 186

Direito humano à alimentação adequada 103, 260

Disseminação da informação 67,73

Distanciamento social 25, 48, 55, 61, 68, 73, 90, 95, 126, 130, 192, 195, 202, 279

Distúrbios psicológicos 190

Doença infecciosa 53, 125, 133, 134, 140, 141, 151, 152

Doença multifatorial 250

Doenças 21, 24, 28, 29, 43, 54, 56, 57, 59, 69, 91, 95, 106, 107, 108, 115, 116, 117, 119, 120, 129, 133, 137, 140, 142, $149,161,163,164,167,171,172,173,177,185,186,193,194,195,207,210,217,222,223,227,250,271$, $272,273,278,279$

Doenças crônicas 105, 171, 218, 221, 227, 273

Doenças negligenciadas 140, 142

\section{$\mathbf{E}$}

Educação em saúde 19, 24, 43, 46, 48, 50, 67, 69, 73, 105, 108, 133, 134, 135, 136, 137, 145, 179, 185, 215, 216, 217, $227,228,238,240,248,271,272,274$

Efeitos colaterais $28,30,31,32,33$

Enfermagem 50, 56, 57, 58, 60, 61, 88, 89, 122, 149, 161, 176, 177, 178, 180, 188, 227, 240, 280

Enfermidade epidêmica 238

Enfrentamento da covid-19 18, 19, 20, 23, 24, 25

Epidemiologia 114, 140, 148, 149, 160, 161, 242, 248

Equipamentos de proteção 24, 43, 47, 78, 171, 197

Equipes multiprofissionais $52,54,55,56,59,60,61,62$

Estratégia saúde da família (esf) 18, 19, 23, 54

Estratégias nutricionais 105

Estresse 77, 78, 81, 82, 83, 86, 87, 88, 89, 94, 172, 190, 192, 193, 197, 206, 207, 209, 210, 211

Etiologia autoimune 250

Evidências científicas 29, 52, 56, 57, 59, 61, 62, 63, 76, 79, 99, 101, 190

F

Feiras agroecológicas 260, 261, 264

Ferramenta tecnológica de saúde 215

Fortalecimento da saúde púbica 67

Frequência cardíaca 230

G

Gestação 52, 53, 54, 57, 58, 59, 61, 62, 63, 224

Glândulas exócrinas 250, 251, 252, 253

Guia alimentar 105, 107, 108, 112, 113

$\mathbf{H}$

Hábitos alimentares 90, 92, 94, 95, 111, 112

Hanseníase 124, 125, 126, 127, 128, 130, 131, 133, 134, 135, 136, 137, 138, 139, 140, 141, 142, 143, 144, 145, 146, $147,148,149$

Herança genética 115

Hipertensão 57, 95, 105, 107, 108, 114, 115, 116, 118, 119, 120, 121, 181, 183, 186, 228, 229, 274, 276,278 
Hiposecreção lacrimal 250

I

Idosos 19, 21, 23, 54, 105, 108, 134, 137

Impacto da pandemia 99

Imunização 28, 29, 30, 35, 38, 179, 183, 186

Inclusão 20, 22, 52, 55, 56, 58, 75, 77, 79, 101, 163, 165, 167, 215, 226, 268

Infecções 28, 29, 38, 43, 44, 48, 57, 59, 60, 78, 165, 172, 180, 227, 251

Infecções respiratórias virais $43,44,48$

Informação de qualidade 271, 279

Informação em saúde 67,69

Informação sobre a hanseníase 124

Instabilidade econômica 99, 103

Instrumento de prevenção 43

Intervenção psicológica 202

Isolamento $23,48,55,60,62,81,90,91,92,94,100,142,164,177,179,190,191,192,193,194,195,196,197,198$, $199,203,204,205,207,208,210,242,261$

Isolamento social 23, 26, 62, 90, 92, 94, 100, 142, 164, 177, 179, 190, 191, 192, 194, 195, 196, 197, 198, 203, 205, 207, 208, 210, 242

$\mathbf{J}$

Janssen (johnson \& johnson) 28, 29, 30

$\mathbf{L}$

Lesões cutâneas 140, 143

Linha de frente 76, 78, 79, 81, 84, 86, 87, 88, 171, 195, 197, 202, 206, 207, 208, 210

M

Má qualidade do sono 77,87

Máscaras faciais 43

Material digital 43, 46

Medição biométrica 230

Medidas de isolamento 26, 28, 194

Medidores de glicose 230

Metodologia pico (problema, intervenção, contexto, resultado) 76, 79, 99, 101

Monitores do centro de ciências da saúde (ccs) 238

Monitoria 238, 239, 240, 241, 242, 243, 244, 246

Morbidade 115, 119

Mortalidade materna 163, 171, 172

Mudanças nos estilos de vida 90, 92, 93

Mycobacterium leprae 135, 140, 141, 146

N

Níveis socioeconômicos 43, 46

Novas modalidades de interações 238

Novo coronavírus sars-cov-2 18, 19 
Olho seco 250, 251, 252, 253, 254, 255, 256, 257, 258

Organização mundial de saúde (oms) 19, 52, 53, 54, 68, 92, 191

Organização pan-americana de saúde (opas) 52, 54

Órgãos oficiais de saúde 238, 247

Outubro rosa $177,178,179,184,185$

$\mathbf{P}$

Padrões dietéticos 115

Pandemia 18, 19, 20, 21, 23, 24, 25, 26, 28, 31, 38, 45, 47, 48, 53, 55, 68, 69, 73, 75, 76, 78, 79, 81, 82, 84, 86, 87, 88, $89,90,91,92,93,94,95,96,99,100,101,102,103,104,117,118,119,120,124,126,130,131,133,135,136$, $137,142,152,153,160,161,162,163,164,165,168,169,170,171,172,173,174,177,179,180,186,187$, 190, 191, 192, 193, 194, 195, 196, 197, 198, 199, 200, 203, 204, 205, 206, 207, 208, 210, 211, 212, 213, 238, $239,240,241,242,244,247,248,271,272,279,280$

Perfil alimentar 105

Perfil clínico 160, 177, 183, 185

Pfizer (pfizer e biontech) 28, 30

Políticas públicas de saúde 18, 24, 173, 204

Portadores de síndrome metabólica 115

Prática oftalmológica 250

Práticas do autocuidado 178,186

Pressão arterial 115,230

Prevenção 29, 33, 72, 170, 187, 221

Primeiros socorros 215, 218, 219, 225, 227, 273

Princípio ativo 28, 30, 33

Produção de alimentos 260

Profissionais de psicologia 202

Profissionais de saúde 18, 22, 24, 54, 56, 76, 78, 79, 81, 82, 84, 85, 86, 87, 89, 107, 125, 138, 151, 160, 170, 185, 194, $195,197,198,202,206,207,208,238$

Programa nacional de alimentação escolar (pnae) 99, 265

Promoção em saúde 43, 45, 271, 278

Propagação do vírus $43,47,48,67,69$

Q

Qualidade de vida 22, 47, 82, 106, 112, 155, 217, 226, 244, 250, 251, 254, 272, 278

Quarentena 90, 100, 121, 126, 170, 172, 190, 191, 192, 193, 194, 195, 199, 202, 206

$\mathbf{R}$

Recurso tecnológico 230

Redes sociais 43, 45, 46, 49, 133, 137, 194, 271, 272, 274, 278, 279

Responsabilidade individual e coletiva 18, 24

Restrição social 90, 92, 93 
Sars-cov- $20,21,29,30,31,32,33,34,35,38,39,40,45,49,53,56,57,58,59,60,65,88,120,163,164,165,168$, $173,191,199,238,239,240,242,243,247,248,279$

Saúde da família 18, 19, 20, 22, 23, 24, 25, 26, 52, 54, 55, 62, 64, 125, 135, 179, 187

Saúde da mulher 163, 166, 173

Saúde de maneira remota 133

Saúde dos profissionais $76,79,81,86,89$

Saúde dos trabalhadores $77,86,87$

Saúde integral 52, 163, 165, 167, 171

Saúde mental 78, 83, 88, 90, 93, 94, 95, 96, 172, 179, 190, 191, 192, 193, 194, 195, 196, 197, 198, 202, 203, 204, 206, $207,208,210,212,222,227,279$

Saúde psicológica 77,87

Saúde pública $6,20,28,38,69,73,82,120,125,134,139,141,148,149,160,168,169,170,171,172,177,179,197$, $199,208,211,238,240,242,247$

Secura ocular 250, 251, 257

Sedentarismo 94, 107, 115, 179

Segurança alimentar e nutricional 99, 100, 101, 102, 103, 104

Segurança alimentar nutricional (san) 99

Serviço de saúde 71, 115, 120, 180

Síndrome de sjögren (ss) 250, 251

Síndrome metabólica 115, 116, 117, 118, 119, 120

Sistema imunológico 58, 115, 117, 120

Sistema nacional de agravos de notificação (sinan) 140, 143

Situação de vulnerabilidade 90, 94, 95, 103

Superfície ocular 250, 254, 258

T

Tecnologia 66, 67, 69, 70, 137, 211, 231, 244

Tecnologias de informação e comunicação 208, 230

Teleatendimento 202, 204

Telemedicina 133, 170, 230, 232

Telessaúde 230, 236

Tempos pandêmicos 271

Terapêutica multidisciplinar 190

Teste de schirmer 250, 255

Testes oftalmológicos 250

Transmissão de informações 271, 279

Transtorno de ansiedade generalizada 190, 192

Tuberculose (tb) 151,152

$\mathbf{U}$

Unidade de saúde da família (usf) 52, 55

Uso das máscaras 43, 45, 48 
Vacina 28, 31, 32, 33, 34, 37, 39, 40, 70, 72, 159, 161, 183, 184, 186, 221

Variantes $28,30,31,34,35,38,49,78$

Vídeos educativos 215, 226

Violência contra a mulher $163,169,170,173,174$

$\mathbf{X}$

Xeroftalmia 250,251 


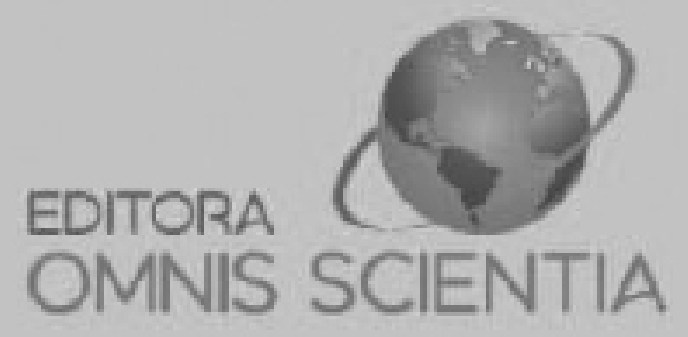

editoraomnisscientia@gmail.com M https://editoraomnisscientia.com.br/ @editora_omnis_scientia (0) https://wwww.facebook.com/omnis.scientia.9 $\boldsymbol{\oplus}$ +55 (87) 9656-3565 (C) 


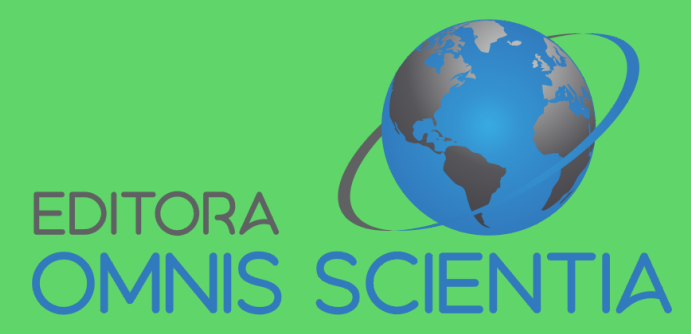

editoraomnisscientia@gmail.com M https://editoraomnisscientia.com.br/ (-) @editora_omnis_scientia (0) https://www.facebook.com/omnis.scientia.9 $\subsetneq$ +55 (87) 9656-3565 @ 